\title{
Double band inversion in $\alpha$-Sn: Appearance of topological surface states and the role of orbital composition
}

\author{
Victor A. Rogalev, ${ }^{1}$ Tomáš Rauch, ${ }^{2}$ Markus R. Scholz, ${ }_{1}^{1}$ Felix Reis, ${ }^{1}$ Lenart Dudy, ${ }^{1}$ Andrzej Fleszar, ${ }^{3}$ \\ Marius-Adrian Husanu, ${ }^{4,5}$ Vladimir N. Strocov, ${ }^{4}$ Jürgen Henk, ${ }^{2}$ Ingrid Mertig, ${ }^{2,6}$ Jörg Schäfer, ${ }^{1}$ and Ralph Claessen ${ }^{1}$ \\ ${ }^{1}$ Physikalisches Institut und Röntgen Center for Complex Material Systems, Universität Würzburg, 97074 Würzburg, Germany \\ ${ }^{2}$ Institute of Physics, Martin Luther University Halle-Wittenberg, 06099 Halle (Saale), Germany \\ ${ }^{3}$ Institut für Theoretische Physik und Astronomie, Universität Würzburg, 97074 Würzburg, Germany \\ ${ }^{4}$ Swiss Light Source, Paul Scherrer Institute, CH-5232 Villigen, Switzerland \\ ${ }^{5}$ National Institute of Materials Physics, 077125, Magurele, Romania \\ ${ }^{6}$ Max Planck Institute for Microstructure Physics, 06120 Halle (Saale), Germany
}

(Received 12 December 2016; published 27 April 2017)

\begin{abstract}
The electronic structure of $\alpha$-Sn (001) thin films strained compressively in-plane was studied both experimentally and theoretically. A new topological surface state (TSS) located entirely within the gapless projected bulk bands is revealed by ab initio-based tight-binding calculations as well as directly accessed by soft $\mathrm{x}$-ray angle-resolved photoemission. The topological character of this state, which is a surface resonance, is confirmed by unravelling the band inversion and by calculating the topological invariants. In agreement with experiment, electronic structure calculations show the maximum density of states in the subsurface region, while the already established TSS near the Fermi level is strongly localized at the surface. Such varied behavior is explained by the differences in orbital composition between the specific TSS and its associated bulk states, i.e., their hybridization, respectively.
\end{abstract}

DOI: 10.1103/PhysRevB.95.161117

Since the theoretical prediction of topological insulators (TIs) $[1,2]$, many materials were found to belong to this new class of solids $[3,4]$. The interest in these materials is based on the idea to exploit and to manipulate spin-polarized metallic surface states with forbidden backscattering due to timereversal symmetry. These so-called topological surface states (TSSs) are induced at an interface between systems that belong to different topological phases. The topologically nontrivial low temperature phase of $\mathrm{Sn}(\alpha-\mathrm{Sn})$ is a particularly interesting example for such a system. In contrast to other TIs, like the $\mathrm{Bi}_{2} \mathrm{X}_{3}$ compounds, in which the inverted bands are separated by a global band gap [5], unstrained $\alpha-\mathrm{Sn}$ is a semimetal [6,7] with diamond structure and the Fermi energy $\left(E_{\mathrm{F}}\right)$ pinned to the $\Gamma_{8}^{+}$level (Fig. 1). Spin-orbit coupling (SOC) shifts the $p$ derived split-off $\Gamma_{7}^{+}$band below the $s$-derived $\Gamma_{7}^{-}$band which in turn is situated below the fourfold degenerate $p$-derived $\Gamma_{8}^{+}$band $[8,9]$. The latter degeneracy can be lifted by applying strain [Fig. 1(b)]. The key feature of topological materials- the band inversion-occurs in $\alpha$-Sn between the $\Gamma_{8}^{+}$and the $\Gamma_{7}^{-}$ levels at the $\Gamma$ point of the Brillouin zone (BZ). Importantly, yet another band inversion exists at the $\Gamma$ point of the BZ within the occupied electronic structure of $\alpha$-Sn: between $\Gamma_{7}^{-}$ and $\Gamma_{7}^{+}$bands, which has gone unnoticed in previous studies.

Although $\alpha$-Sn has been predicted to be topologically nontrivial [10], neither the electronic structure nor the existence of surface states was discussed in detail until the experimental observation of a TSS which emerges between the $\Gamma_{7}^{-}$and $\Gamma_{8}^{+}$levels and exhibits a Dirac point close to $E_{\mathrm{F}}[8,11,12]$; we refer to this state as TSS1 from now on. Interestingly, although TSS1 is largely degenerate with the surface-projected bulk $\Gamma_{8}^{+}$band, in angle-resolved photoelectron spectroscopy (ARPES) it is detected as a sharply defined dispersive peak [Fig. 1(c)]. Moreover, a very recent experimental report on spin-to-charge conversion supposedly involving the TSS1 of
$\alpha$-Sn [12] demonstrates a practical utility of this material for spintronics. The above results raise two questions: What is the mechanism that protects TSS1 from hybridization with the bulk states, and can we expect another TSS (TSS2) induced by the other band inversion?

Here we report on a new surface state in the occupied electronic structure of strained $\alpha$-Sn (001) films, explored in a combined theoretical and experimental approach. Electronicstructure calculations reveal that this state bridges the $\Gamma_{7}^{-}$and $\Gamma_{7}^{+}$bands and is located in the subsurface region, thus penetrating deeper into the bulk than TSS1 $[8,11]$. Bulk-sensitive soft X-ray ARPES (SX-ARPES) provides direct experimental evidence for this new subsurface state, which has been overlooked in previous low-photon-energy ARPES experiments with smaller probing depths. Analyzing the orbital characters of bulk and surface states, we show that the different decay character of two surface states is explained by hybridization with the corresponding bulk states. In particular, TSS1's orbital composition suppresses hybridization with surface-projected bulk states, while for the new surface state such hybridization is favored. Moreover, we review the topological properties of the bulk-band structure by calculating the $\mathcal{Z}_{2}$ invariant as well as the mirror Chern numbers. We find that the new 'buried' surface state is topologically nontrivial despite being located further away from the topological phase discontinuity (interface TI/vacuum).

Thin films of $\alpha$-Sn were grown in situ on $\operatorname{InSb}(001)$ substrates. Prior to deposition, substrates have been cleaned in a series of sputter-anneal cycles, while the surface quality was monitored by low-energy electron diffraction (see Ref. [13]). $\mathrm{Sn}$ was deposited on the substrates at room temperature; simultaneously Te was deposited to $n$-dope the film and to provide a smooth growth [8]. The thickness of the $\alpha$-Sn film was at least $\approx 6 \mathrm{~nm}$ (9 unit cells) as estimated from 
(a)

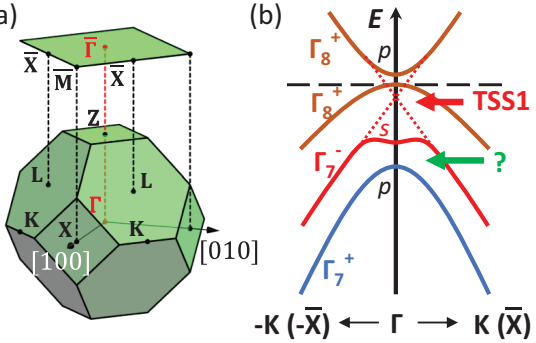

(c)

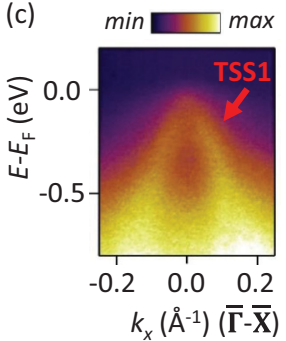

FIG. 1. Brillouin zone and electronic structure of strained $\alpha$-Sn (001). (a) Bulk and surface BZ. (b) Sketch of the band structure along $\Gamma \mathrm{KX}(\bar{\Gamma} \overline{\mathrm{X}})$ assuming in-plane (001) strain and SOC. Band parities are encoded by + or - superscripts, while dominant orbital compositions near the $\Gamma$ point ( $p$ or $s$ ) identify two band inversions (indicated by red and green arrows). (c) ARPES data taken with the He-I line $(h v=21.2 \mathrm{eV})$ show TSS1 near $E_{\mathrm{F}}$.

photoemission data [13]. SX-ARPES measurements have been performed at the ADRESS beamline of the Swiss Light Source. Details of the experimental setup are given in Ref. [14]. While bulk $\alpha$-Sn is stable only below $\approx 13.2^{\circ} \mathrm{C}[15]$, thin films of $\alpha$-Sn epitaxially deposited on $\mathrm{InSb}(001)$ substrates are stable up to $\approx 200^{\circ} \mathrm{C}$ [16], which guarantees successful growth at room temperature.

The InSb substrate induces compressive strain in $\alpha$-Sn due to the lattice mismatch of $0.13 \%$, the latter causing a local band gap of $\approx 30 \mathrm{meV}$ at the $\Gamma$ point of the bulk Brillouin zone. Similar to HgTe $[17,18]$, a global band gap in the entire reciprocal space is opened only by tensile in-plane (001) strain, whereas for compressive in-plane (001) strain the local band gap around the bulk $\Gamma$ point closes along the $\Gamma-\mathrm{Z}$ direction $[13,19]$. The resulting band crossing gives rise to a Dirac point in the bulk band structure. These properties suggest that compressively strained $\alpha-\mathrm{Sn}$ is a Dirac semimetal. We note that the closing of the band gap does not alter the topological character [8,11]: The former occurs between bands whose associated states have even parity [Fig. 1(b)].

Both band inversions in $\alpha$-Sn can be understood from the orbital composition of the bulk bands, see Fig. 1(b) and Fig. S2 of Ref. [13]. The upper band inversion is a consequence of the $\Gamma_{7}^{-} s$-derived level being located below the $p$-derived $\Gamma_{8}^{+}$ level, contrary to ordinary semiconductors. The lower band inversion in turn originates from SOC, which pushes the $\Gamma_{7}^{+}$ spin-orbit split level below $\Gamma_{7}^{-}$. We note that a similar bulk bands arrangement with two band inversions, i.e., $p$-derived $\Gamma_{8}>s$-derived $\Gamma_{6}>$ spin-orbit split $\Gamma_{7}$, can be also found in $\mathrm{HgTe}$ [20]. In fact, an analogous twofold inverted band order has recently been reported for several semiconductor families, ranging from ternary Heusler compounds [21,22] to III-V narrow gap semiconductor alloys like $\operatorname{InAs}_{x} \mathrm{Sb}_{1-x}$ under tensile (biaxial) strain of $2-5 \%$ [23,24].

To explore the bulk and surface electronic structure of $\alpha$-Sn we first performed $a b$ initio-based tight-binding (TB) calculations for bulk and semi-infinite systems. The Slater-Koster parameters $[13,25]$ for first- and second-nearest neighbors as well as the spin-orbit coupling strength were obtained by optimizing the TB band structure with respect to $a b$ initio data $[6,9]$. Special attention has been paid to reproducing the correct band ordering near the $\Gamma$ point.
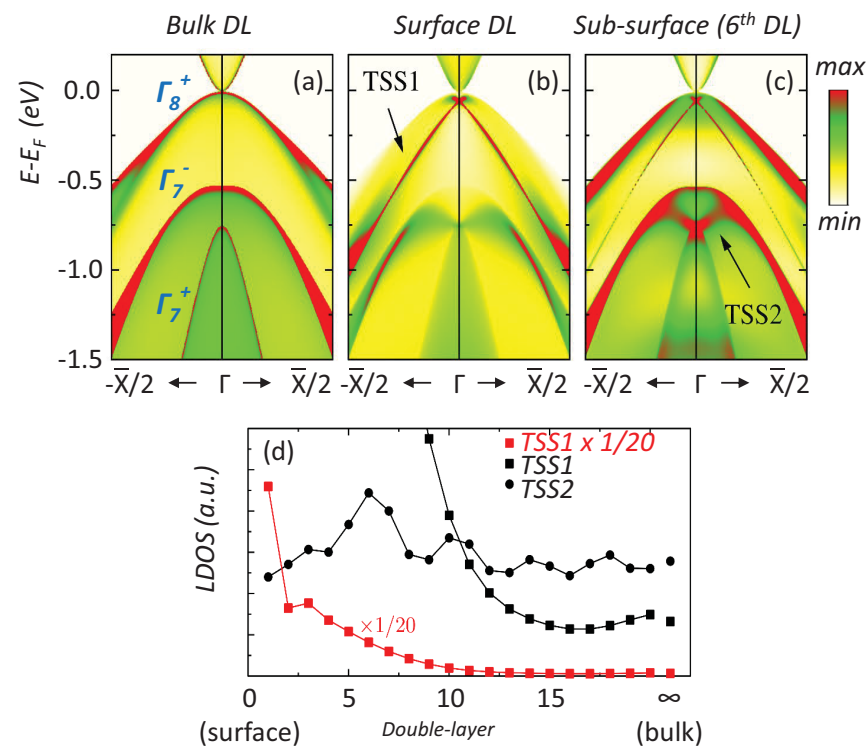

FIG. 2. Theoretical electronic structure of $\alpha$-Sn (001) obtained by TB calculations. The spectral density, given by color scale, is depicted for (a) a bulk double layer (DL), (b) a surface DL, and (c) the sixth subsurface DL. (d) Layer-resolved spectral density near $\bar{\Gamma}$ for TSS1 (red and black squares) and TSS2 (black circles). While TSS1 is surface localized, TSS2 is located in the subsurface region, with an LDOS maximum in the sixth DL. Red and black squares show identical but differently scaled data for TSS1.

The TB results are presented in Fig. 2 (see also Ref. [13]), where we show a depth distribution of the electronic states' wave functions. When projected onto a Sn double layer (DL; half of the cubic unit cell) within the bulk [Fig. 2(a)], the $\Gamma_{8}^{+}$, $\Gamma_{7}^{-}$, and $\Gamma_{7}^{+}$bands remain unconnected, in agreement with the bulk electronic structure [6]. On the contrary, when projected onto the DL near the surface [Figs. 2(b) and 2(c)], TSS1 shows up as a consequence of the $\Gamma_{7}^{-} / \Gamma_{8}^{+}$band inversion, exhibiting the maximum spectral density in the topmost DL. Importantly, a new 'connecting' spectral density located between the $\Gamma_{7}^{-}$ and $\Gamma_{7}^{+}$bulk bands appears at $\approx-0.8 \mathrm{eV}$ [Fig. 2(c)]; we refer to this feature as TSS2 in the following. The spectral density of TSS2 is enhanced in the subsurface region, having an extended maximum at and around the sixth DL, as shown in Fig. 2(d). Although the dispersive behavior may at first glance look like a Rashba split $\Gamma_{7}^{-}$band, such an explanation can be ruled out, as its overall band width does not change from surface to bulk, whereas a Rashba effect would occur only at the surface and inevitably increase the width of the $\Gamma_{7}^{-}$band. Based on our slab calculations [13] a quantum well origin of this state can be excluded as well.

We note that the so far undiscovered TSS2 exists in an 'inverted' SOC-induced band gap, which suggests a topological origin [Fig. 1(b)]. This conjecture and, thus, the topological nontrivial band ordering are affirmed by calculations of topological invariants. Originally defined for insulating systems [26], the $\mathcal{Z}_{2}$ invariant has also been calculated for systems without a global band gap $[27,28]$. A necessary condition for such calculations is the presence of a finite energy difference at each wave vector $\vec{k}$ (i. e., a $\vec{k}$-dependent band gap). This is the case for $\alpha-\mathrm{Sn}$ in which the $\Gamma_{7}^{+}$band is separated from 
the $\Gamma_{7}^{-}$band in the entire bulk BZ, irrespectively whether moderate compressive or tensile strain is applied (strain less than $3 \%$ is assumed). The $\mathcal{Z}_{2}$ invariant of the bands below the $\Gamma_{7}^{-}$band was calculated to $\left(v_{0} ; v_{1} v_{2} v_{3}\right)=(1 ; 000)$ by using the Fu-Kane formula [29] discretized by Fukui and Hatsugai [27] and by tracing maximally localized Wannier functions [30]. Moreover, the lattice is invariant upon reflection at the (110) and the (1) $\overline{10}$ ) mirror planes; the respective mirror Chern numbers are $n_{\mathrm{M}}=-1$ for both planes (further details in the Supplemental Material [13]). Both topological invariants confirm the nontrivial topology: Moderately strained $\alpha$-Sn is both a strong TI and a topological crystalline insulator. This necessitates that according to the bulk-boundary correspondence [3] a TSS has to bridge ('interconnect') the $\Gamma_{7}^{+}$and $\Gamma_{7}^{-}$ bands: This state is unambiguously identified as TSS2.

The absence of both TSS1 and TSS2 in the bulk electronic structure [Fig. 2(a)] is clear evidence for their surface character. It is also evident from comparing Fig. 2(b) with Fig. 2(c) that TSS1 has its LDOS maximum at the surface while TSS2 is located well below the surface. The different surface localization is addressed in Fig. 2(d). Due to overlap with projected bulk bands, both TSS1 and TSS2 are strictly speaking so-called 'surface resonances,' which are characterized by an enhanced LDOS in the surface or subsurface region being degenerate with a finite LDOS in the bulk. Yet TSS1 decays in general exponentially toward the bulk, thereby being more reminiscent of a surface state; in contrast, the LDOS of TSS2 decays and oscillates, which is more typical of a surface resonance.

To probe the subsurface-localized TSS2 experimentally we utilized SX-ARPES which has a higher probing depth compared to low-photon-energy ARPES [31]. Owing to an excellent $k_{\perp}$ resolution, the experimental SX-ARPES data acquired with polarized $352 \mathrm{eV}$ and $684 \mathrm{eV}$ photons detect mostly states close to the bulk $\Gamma$ points [Fig. 3(a)]; they show clearly all three occupied bulk valence bands, i.e., $\Gamma_{8}^{+}$, $\Gamma_{7}^{-}$, and $\Gamma_{7}^{+}$[Figs. 3(b) and 3(c)]. While at both photon energies the intensity from TSS1 near $E_{\mathrm{F}}$ is very weak, TSS2 located between the surface-projected $\Gamma_{7}^{-}$and $\Gamma_{7}^{+}$bulk bands at $\approx-0.8 \mathrm{eV}$ is unequivocally resolved [Figs. 3(e) and 3(f)]; it is strikingly similar to TSS2 in the calculations [Fig. 2(c)]. The fact that along the surface perpendicular [001] direction TSS2 appears only near bulk $\Gamma$ points and persists in spectra acquired with high probing depth $(h v=684 \mathrm{eV})$ clearly indicates its surface resonance character. The apparent broadening of the $\Gamma_{7}^{+}$band at $h v=352 \mathrm{eV}$ is most likely a consequence of a small yet finite $k_{\perp}$ resolution [32].

While TSS 1 appears very pronounced between the $\Gamma_{7}^{-}$and the $\Gamma_{8}^{+}$bands at photon energies around $20 \mathrm{eV}$, the intensity of TSS1 is small at soft X-ray photon energies (see Ref. [13]). Interestingly, in a study by Liu et al. on $\mathrm{HgTe}(110)$ [33], which closely resembles the electronic structure of $\alpha-\mathrm{Sn}$, a counterpart of TSS1 was observed over a wide SX-photon energy range. The small intensity of TSS 1 in $\alpha$-Sn might be better understood by future SX-ARPES photoemission simulations.

As depicted in Fig. 3(d), the $\Gamma_{8}^{+}$band crosses $E_{\mathrm{F}}$, indicating that the film is $p$ doped; this may be explained by $\mathrm{Sn}$ vacancies or diffusion of In atoms from the substrate into the film [8]. We note that the small negative partial band gap between both $\Gamma_{8}^{+}$ bands described above and in Ref. [13] is likely to occur above $E_{\mathrm{F}}$ and is, for the small strain induced through the InSb substrate $(0.13 \%)$, below the resolution limit of our experiment.

In order to better understand the different spatial distribution of the TSS1 and TSS2, we explore their orbital

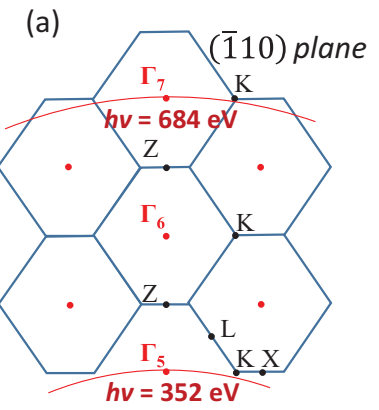

(d)

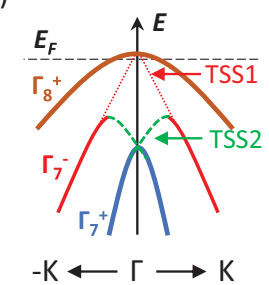

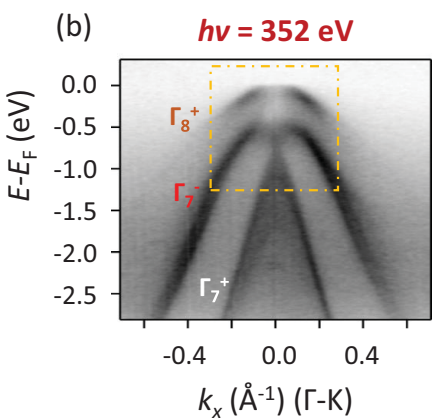

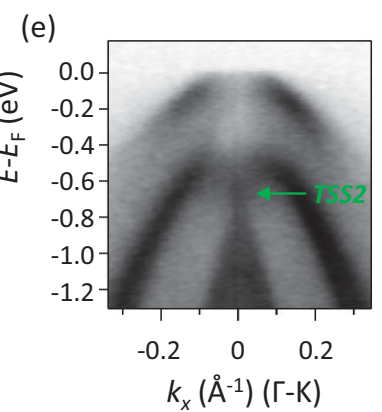

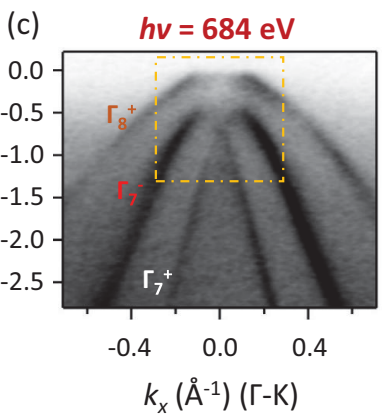

(f)

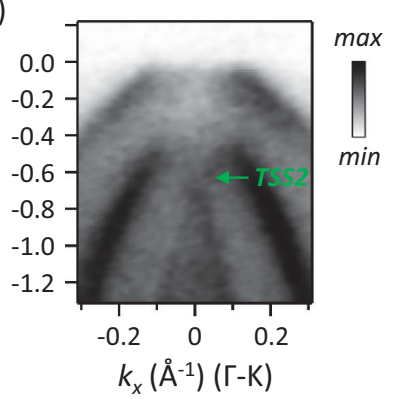

FIG. 3. Experimental electronic structure of $\alpha$-Sn (001). (a) Sketch of the bulk BZ in the (110) mirror plane and ARPES measurement path for two photon energies. (b) ARPES map $I\left(E, k_{x}\right)$ taken for $h v=352 \mathrm{eV}$ and $p$-polarized light along $\Gamma \mathrm{K}(\bar{\Gamma} \overline{\mathrm{X}})$. (c) As (b) but $h v=684 \mathrm{eV}$ and for circular polarized light. (d) Sketch of the observed dispersions based on the data shown in (b) and (c). (e) and (f) Zoom into regions shown with yellow frames on (b) and (c). Although the intensity of TSS1 is weak, bulk bands and TSS2 are clearly resolved. TSS1 becomes more visible in a second derivative of the ARPES map $I\left(E, k_{x}\right)[13]$. 

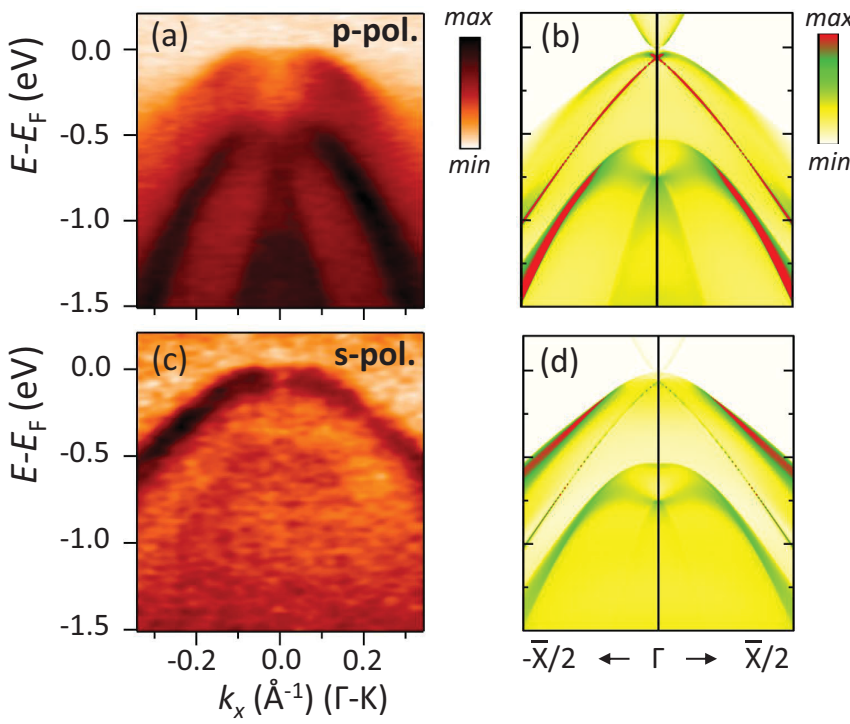

FIG. 4. Orbital composition of the electronic states. (a) ARPES map $I\left(E, k_{x}\right)$ measured along $\Gamma \mathrm{K}(\bar{\Gamma} \overline{\mathrm{X}})$ for $h v=352 \mathrm{eV}$ and $p$ polarized light. The main contribution comes from electronic states that are symmetric w. r. t. the $\mathrm{X} \Gamma \mathrm{Z}$ mirror plane. (b) Computed spectral density of symmetric orbitals (summed over first six DLs). (c) As (a) but for $s$-polarized light. High intensities are due to antisymmetric states. (d) As (b) but for antisymmetric orbitals.

composition using two distinct polarizations of the incident light (Fig. 4). In the experimental geometry, the measurement plane coincides with a ( $\overline{1} 10)$ mirror plane $(\mathrm{X} Г \mathrm{Z})$. Therefore, for light polarized parallel (even) to the measurement plane ( $p$ polarized light), photoemission from initial states whose wave function is antisymmetric (odd) with respect to the $(\overline{1} 10)$ mirror plane is suppressed, since the final state of the photoelectron is even [34]. Thus, only states with dominant $s, p_{z}$, and symmetric combinations of $p_{x}$ and $p_{y}$ orbitals are visible in Fig. 4(a). In turn, with perpendicular polarization (odd or $s$-polarized light) one detects states with antisymmetric wave functions [i. e., antisymmetric combinations of $p_{x}$ and $p_{y}$ orbitals; Fig. 4(c)]. To explain the experimental polarization dependence, we computed orbital-resolved spectral densities; only states symmetric or antisymmetric to the (110) plane summed over the first six DLs are shown in Figs. 4(b) and 4(d). The results fit very well to the experimental SX-ARPES data and provide a strong hint towards a similar orbital composition of TSS2 and the projected bulk states.

Further investigations of the orbital compositions show that the projected bulk states between the $\Gamma_{7}^{+}$and $\Gamma_{7}^{-}$ levels are mainly composed of $p_{x}$ and $p_{y}$ orbitals but lack $p_{z}$ contributions (Fig. S5 in Ref. [13]). The same orbital composition is found for TSS2, which explains the high degree of its hybridization with the bulk states and its pronounced surface resonance character. On the contrary, TSS 1 is mainly composed of $p_{z}$ orbitals; it therefore hybridizes much weaker with the $p_{x}$ and $p_{y}$ bulk states below $\Gamma_{8}^{+}$, which results in its exponential decay toward the bulk.

Bearing resemblance to Weyl or Dirac semimetals in which both topological bulk and surface states cross the Fermi level [17-19,35-37], TSSs can appear degenerate with the surfaceprojected bulk band structure as long as they reside within local energy- and wave-vector-dependent band gaps [27,28,38,39]. Moreover, such states were observed to appear also in spinorbit induced partial gaps away from the $E_{\mathrm{F}}$ in well-known layered topological insulators, e.g., $\mathrm{Sb}_{2} \mathrm{Te}_{3}[40,41]$ and $\mathrm{Bi}_{2} \mathrm{Se}_{3}$ $[42,43]$. However, a pair of surface-resonance TSSs which share the same bulk band and have very different spatial decay character is so far a unique property of $\alpha-\mathrm{Sn}$.

In conclusion, the surface and subsurface electronic structure of $\alpha$-Sn thin films strained compressively in the (001) plane is studied theoretically and experimentally. In agreement with $a b$ initio-based electronic structure calculations, our experimental data allow us to reveal an additional topological surface state. The nontrivial topology of this surface state, which is a consequence of a SOC-induced band inversion, is proven by the topological invariants of the associated bulk states. This new TSS of $\alpha$-Sn in the projected valence bands has a pronounced surface resonance character, in clear contrast to the surface-localized TSS1, which is attributed to the different degree of hybridization with surface-projected bulk states. Our findings thus reveal a new type of TSS in $\alpha$-Sn that is coupled by orbital symmetries to the bulk states in the whole energy range. We emphasize that bulk band inversions similar to $\alpha$-Sn can be found also in HgTe [20], some ternary Heusler compounds [21,22], and biaxially strained $\operatorname{InAs}_{x} \mathrm{Sb}_{1-x}[23,24]$. This suggests that TSS 1 in these materials could have a similar character as in $\alpha$-Sn, and a surface-resonant TSS 2 could occur due to a second band inversion. Recently, a pair of surface states reminiscent of TSS1 and TSS2 discussed in the present work was observed in half-Heusler $\mathrm{RPtBi}(\mathrm{R}=\mathrm{Lu}, \mathrm{Gd})$ [44] and PtLuSb [45] compounds. The authors of those studies attribute a nontrivial topology to the surface state near $E_{\mathrm{F}}$; considering our findings one may speculate that the other surface state is of topological origin, too.

This work was supported by Collaborative Research Center (CRC) 1170 ToCoTronics and Priority Program SPP 1666 of the Deutsche Forschungsgemeinschaft (DFG). A.F. thanks the Deutsche Forschungsgemeinschaft for support (Research Unit FOR 1162) and the Jülich Supercomputing Centre for providing the computer resources (project No. hwb03). We thank J. Minár and J. Braun for sharing and discussing the results of the photoemission calculations on $\alpha$-Sn. We are also thankful to $\mathrm{G}$. Li for promotive discussions.
[1] C. L. Kane and E. J. Mele, Phys. Rev. Lett. 95, 146802 (2005).

[2] C. L. Kane and E. J. Mele, Phys. Rev. Lett. 95, 226801 (2005).

[3] M. Z. Hasan and C. L. Kane, Rev. Mod. Phys. 82, 3045 (2010).

[4] A. Bansil, H. Lin, and T. Das, Rev. Mod. Phys. 88, 021004 (2016).
[5] H. Zhang, C.-X. Liu, X.-L. Qi, X. Dai, Z. Fang, and S.-C. Zhang, Nat. Phys. 5, 438 (2009).

[6] T. Brudevoll, D. S. Citrin, M. Cardona, and N. E. Christensen, Phys. Rev. B 48, 8629 (1993).

[7] T. G. Pedersen, C. Fisker, and R. V. Jensen, J. Phys. Chem. Solids 71, 18 (2010). 
[8] A. Barfuss, L. Dudy, M. R. Scholz, H. Roth, P. Höpfner, C. Blumenstein, G. Landolt, J. H. Dil, N. C. Plumb, M. Radovic, A. Bostwick, E. Rotenberg, A. Fleszar, G. Bihlmayer, D. Wortmann, G. Li, W. Hanke, R. Claessen, and J. Schäfer, Phys. Rev. Lett. 111, 157205 (2013).

[9] S. Küfner, J. Furthmüller, L. Matthes, M. Fitzner, and F. Bechstedt, Phys. Rev. B 87, 235307 (2013).

[10] L. Fu and C. L. Kane, Phys. Rev. B 76, 045302 (2007).

[11] Y. Ohtsubo, P. Le Fèvre, F. Bertran, and A. Taleb-Ibrahimi, Phys. Rev. Lett. 111, 216401 (2013).

[12] J.-C. Rojas-Sánchez, S. Oyarzún, Y. Fu, A. Marty, C. Vergnaud, S. Gambarelli, L. Vila, M. Jamet, Y. Ohtsubo, A. Taleb-Ibrahimi, P. Le Fèvre, F. Bertran, N. Reyren, J.-M. George, and A. Fert, Phys. Rev. Lett. 116, 096602 (2016).

[13] See Supplemental Material at http://link.aps.org/supplemental/ 10.1103/PhysRevB.95.161117 for details on sample characterization, semi-infinite tight-binding calculations, double band inversion, calculation of topological invariants, effects of strain on the bulk band structure, orbital composition of states, and additional experimental data, which includes Refs. [46-54].

[14] V. N. Strocov, M. Kobayashi, X. Wang, L. L. Lev, J. Krempasky, V. V. Rogalev, T. Schmitt, C. Cancellieri, and M. L. ReinleSchmitt, Synchrotron Radiation News 27, 31 (2014).

[15] A. Jayaraman, W. Klement, and G. C. Kennedy, Phys. Rev. 130, 540 (1963).

[16] B. Mason and B. Williams, Surf. Sci. 273, L472 (1992).

[17] T. Rauch, S. Achilles, J. Henk, and I. Mertig, Phys. Rev. Lett. 114, 236805 (2015)

[18] J. Ruan, S.-K. Jian, H. Yao, H. Zhang, S.-C. Zhang, and D. Xing, Nat. Commun. 7, 11136 (2016)

[19] B.-J. Yang and N. Nagaosa, Nat. Commun. 5, 4898 (2014).

[20] B. A. Bernevig, T. L. Hughes, and S.-C. Zhang, Science 314, 1757 (2006).

[21] S. Chadov, X. Qi, J. Kübler, G. H. Fecher, C. Felser, and S. C. Zhang, Nat. Mater. 9, 541 (2010).

[22] H. Lin, L. A. Wray, Y. Xia, S. Xu, S. Jia, R. J. Cava, A. Bansil, and M. Z. Hasan, Nat. Mater. 9, 546 (2010).

[23] W. Feng, W. Zhu, H. H. Weitering, G. M. Stocks, Y. Yao, and D. Xiao, Phys. Rev. B 85, 195114 (2012).

[24] S. Namjoo, A. S. H. Rozatian, and I. Jabbari, J. Alloys Compd. 628, 458 (2015)

[25] J. C. Slater and G. F. Koster, Phys. Rev. 94, 1498 (1954).

[26] L. Fu, C. L. Kane, and E. J. Mele, Phys. Rev. Lett. 98, 106803 (2007).

[27] T. Fukui and Y. Hatsugai, J. Phys. Soc. Jpn. 76, 053702 (2007).

[28] J. C. Y. Teo, L. Fu, and C. L. Kane, Phys. Rev. B 78, 045426 (2008).

[29] L. Fu and C. L. Kane, Phys. Rev. B 74, 195312 (2006).

[30] R. Yu, X. L. Qi, A. Bernevig, Z. Fang, and X. Dai, Phys. Rev. B 84, 075119 (2011).

[31] S. Tanuma, C. J. Powell, and D. R. Penn, Surf. Interface Anal. 21, 165 (1994).

[32] V. N. Strocov, J. Electron Spectrosc. Relat. Phenom. 130, 65 (2003).

[33] C. Liu, G. Bian, T. R. Chang, K. Wang, S. Y. Xu, I. Belopolski, I. Miotkowski, H. Cao, K. Miyamoto, C. Xu, C. E. Matt, T. Schmitt, N. Alidoust, M. Neupane, H. T. Jeng, H. Lin, A. Bansil,
V. N. Strocov, M. Bissen, A. V. Fedorov, X. Xiao, T. Okuda, Y. P. Chen, and M. Z. Hasan, Phys. Rev. B 92, 115436 (2015).

[34] J. Hermanson, Solid State Commun. 22, 9 (1977).

[35] D. Bulmash, C. X. Liu, and X. L. Qi, Phys. Rev. B 89, 081106 (2014).

[36] H. Inoue, A. Gyenis, Z. Wang, J. Li, S. W. Oh, S. Jiang, N. Ni, B. A. Bernevig, and A. Yazdani, Science 351, 1184 (2016).

[37] B. Q. Lv, H. M. Weng, B. B. Fu, X. P. Wang, H. Miao, J. Ma, P. Richard, X. C. Huang, L. X. Zhao, G. F. Chen, Z. Fang, X. Dai, T. Qian, and H. Ding, Phys. Rev. X 5, 031013 (2015).

[38] D. Thonig, T. Rauch, H. Mirhosseini, J. Henk, I. Mertig, H. Wortelen, B. Engelkamp, A. B. Schmidt, and M. Donath, Phys. Rev. B 94, 155132 (2016).

[39] D. Kutnyakhov, S. Chernov, K. Medjanik, R. Wallauer, C. Tusche, M. Ellguth, S. A. Nepijko, M. Krivenkov, J. Braun, S. Borek, J. Minár, H. Ebert, H. J. Elmers, and G. Schönhense, Sci. Rep. 6, 29394 (2016).

[40] C. Pauly, G. Bihlmayer, M. Liebmann, M. Grob, A. Georgi, D. Subramaniam, M. R. Scholz, J. Sánchez-Barriga, A. Varykhalov, S. Blügel, O. Rader, and M. Morgenstern, Phys. Rev. B 86, 235106 (2012).

[41] C. Seibel, H. Bentmann, J. Braun, J. Minár, H. Maaß, K Sakamoto, M. Arita, K. Shimada, H. Ebert, and F. Reinert, Phys. Rev. Lett. 114, 066802 (2015).

[42] D. Niesner, T. Fauster, S. V. Eremeev, T. V. Menshchikova, Y. M Koroteev, A. P. Protogenov, E. V. Chulkov, O. E. Tereshchenko, K. A. Kokh, O. Alekperov, A. Nadjafov, and N. Mamedov, Phys. Rev. B 86, 205403 (2012).

[43] J. A. Sobota, S. L. Yang, A. F. Kemper, J. J. Lee, F. T. Schmitt, W. Li, R. G. Moore, J. G. Analytis, I. R. Fisher, P. S. Kirchmann, T. P. Devereaux, and Z. X. Shen, Phys. Rev. Lett. 111, 136802 (2013).

[44] C. Liu, Y. Lee, T. Kondo, E. D. Mun, M. Caudle, B. N. Harmon, S. L. Bud'ko, P. C. Canfield, and A. Kaminski, Phys. Rev. B 83, 205133 (2011).

[45] J. A. Logan, S. J. Patel, S. D. Harrington, C. M. Polley, B. D. Schultz, T. Balasubramanian, A. Janotti, A. Mikkelsen, and C. J. Palmstrøm, Nat. Commun. 7, 11993 (2016).

[46] P. Fantini, M. G. Betti, C. Mariani, E. Magnano, M. Pivetta, and M. Sancrotti, Surf. Sci. 454, 807 (2000).

[47] M. D. Jaffe and J. Singh, Solid State Commun. 62, 399 (1987)

[48] N. Metropolis, A. W. Rosenbluth, M. N. Rosenbluth, A. H. Teller, and E. Teller, J. Chem. Phys. 21, 1087 (1953).

[49] M. Berry, Proc. R. Soc. Lond. A 392, 45 (1984).

[50] W. A. Harrison, Elementary Electronic Structure (World Scientific, New Jersey, 2004).

[51] O. Madelung, U. Rössler, and M. Schulz (eds.) LandoltBörnstein - Group III Condensed Matter (Springer, Berlin, Heidelberg, 2002)

[52] A. Bödicker, W. Schattke, J. Henk, and R. Feder, J. Phys.: Condens. Matter 6, 1927 (1994).

[53] J. Henk and W. Schattke, Comput. Phys. Commun. 77, 69 (1993).

[54] T. Inui, Y. Tanabe, and Y. Onodera, in Group Theory and Its Applications, edited by M. Cardona, P. Fulde, K. von Klitzing, and H.-J. Queisser (Springer-Verlag, Heidelberg, Germany, 1990) 